\title{
Altered White Matter Integrity in Adolescents with Prelingual Deafness: A High-Resolution Tract-Based Spatial Statistics Imaging Study
}

\author{
W. Miao, J. Li, M. Tang, J. Xian, W. Li, Z. Liu, S. Liu, B.A. Sabel, Z. Wang, and H. He
}

\begin{abstract}
BACKGROUND AND PURPOSE: Prelingual deafness is a hearing loss that occurs before language is acquired and may result in brain structural alterations. We studied microstructural WM alterations in prelingually deaf adolescents by using DTI. We hypothesized that any morphologic alterations are mainly located in the auditory association areas. Furthermore, considering that the developing brain is both more vulnerable to deprivation and more plastic than the adult brain, we speculated that the affected areas should be larger than those previously reported in adult deafness.
\end{abstract}

MATERIALS AND METHODS: Diffusion tensor images were obtained from 16 prelingually deaf adolescents (age range, 10-18 years) and 16 healthy control adolescents matched for age and sex. Both groups were compared in fractional anisotropy and radial diffusivity by tract-based spatial statistics. In addition, we examined the correlation between the structural data (FA, RD) differences and the duration of sign language use and hearing aid experience.

RESULTS: Prelingually deaf adolescents had significantly lower FA and increased RD in the bilateral superior temporal gyri, Heschl gyrus, planum polare, and the splenium of the corpus callosum. Only RD values in the right superior temporal gyrus correlated significantly and negatively $(r=$ $-0.518 ; P=.040$ ) with duration of sign language use. These alterations were larger than those previously reported in adult deafness.

CONCLUSIONS: As expected, we found severe morphologic changes of decreased FA and increased RD in multiple auditory association areas and in the corpus callosum. These changes are signs of development impairments in prelingually deaf adolescents, possibly reflecting axonal loss or lack of myelination.

ABBREVIATIONS: FA = fractional anisotropy; $\mathrm{HG}=$ Heschl gyrus; $\mathrm{MD}$ = mean diffusivity; RD = radial diffusivity; STG = superior temporal gyrus; TBSS = tract-based spatial statistics

$\mathbf{P}$ relingual deafness is a profound hearing impairment before the acquisition of language. It typically happens before age 2 years, and the incidence is approximately 1 per 1000 live births. ${ }^{1}$

Received July 9, 2012; accepted after revision August 6.

From the State Key Laboratory of Management and Control for Complex Systems (W.M., W.L., B.A.S., H.H.) and National Lab of Pattern Recognition (M.T.), Institute of Automation, Chinese Academy of Sciences, Beijing, China; Department of Radiology (I.L., J.X., Z.L., Z.W.); and Beijing Institute of Otolaryngology (S.L.), Beijing Tongren Hospital, Capital Medical University, Beijing, China; and Otto-von-Guericke University of Magdeburg, Medical Faculty, Institute of Medical Psychology (B.A.S.), Magdeburg, Germany.

Wen Miao and Jianhong Li contributed equally to this study.

This study was supported by the National Natural Science Foundation of China (No. 61271151, No. 61228103); Beijing Natural Science Foundation (No. 7082026); and Chinese Academy of Sciences Visiting Professorships.

Please address correspondence to Huiguang He, Key Laboratory of Complex Systems and Intelligence Science, Institute of Automation, Chinese Academy of Sciences, Beijing, China, 100190; email: huiguang.he@ia.ac.cn or Junfang Xian, Department of Radiology, Beijing Tongren Hospital, Capital Medical University, Beijing China, 100730; email: cjr.xianjunfang@vip.163.com

- Indicates open access to non-subscribers at www.ajnr.org

http://dx.doi.org/10.3174/ajnr.A3370
Prelingual deafness is either congenital or the result of abnormal brain development, which is acquired by ototoxic medication, bacterial meningitis, or traumatic injury during the first few years of life. Deprivation of sound stimuli before the sensitive period of language development may lead to brain structural reorganization $^{2}$ and change $e^{3,4}$ in prelingually deaf persons. Morphologic correlates have been documented by MR imaging showing that deaf persons have significantly decreased volumes in auditory brain areas or an increased gray matter/WM ratio in the Heschl gyrus and in other auditory-related brain areas. ${ }^{5-8}$ Electroencephalographic studies in children with cochlear implants confirmed the need for proper stimulation in early life. ${ }^{9}$ However, despite treatment, gross alterations in brain anatomy may still prevail as a consequence of prelingual deafness, but the localization and extent of such changes are not well understood. Previous studies ${ }^{10,11}$ identified areas showing relatively small structural changes, which were mainly located in the STG, HG, and corpus callosum. Our hypothesis was that the prelingual deafness-induced WM alterations (locations of significant changes in diffusion tensor imaging 
Table 1: Demographic data of children with prelingual deafness

\begin{tabular}{|c|c|c|c|c|c|c|c|c|}
\hline \multirow[b]{2}{*}{ Patient No } & \multirow[b]{2}{*}{ Age (y) } & \multirow[b]{2}{*}{ Sex } & \multirow[b]{2}{*}{ Hearing Loss (dB) } & \multicolumn{2}{|c|}{ Use of CSL } & \multicolumn{2}{|c|}{ Use of HA } & \multirow[b]{2}{*}{ Cause of Deafness } \\
\hline & & & & Start Age (y) & Duration (y) & Start Age (y) & Duration (y) & \\
\hline 1 & 13 & $\mathrm{~F}$ & 96.5 & 8 & 5 & 6 & 7 & 1 \\
\hline 2 & 16 & $M$ & 118 & 6 & 10 & 6 & 10 & 1 \\
\hline 3 & 18 & $M$ & 100 & 6 & 12 & 6 & 12 & 1 \\
\hline 4 & 16 & $M$ & 107.5 & 6 & 10 & 2 & 14 & 2 \\
\hline 5 & 15 & $\mathrm{~F}$ & 107.5 & 10 & 5 & 10 & 5 & 1 \\
\hline 6 & 13 & $\mathrm{~F}$ & 107.5 & 9 & 4 & 3 & 10 & 2 \\
\hline 7 & 10 & $M$ & 105 & 5 & 7 & 5 & 7 & 2 \\
\hline 8 & 14 & $\mathrm{~F}$ & 110 & 3 & 11 & 1.5 & 12.5 & 2 \\
\hline 9 & 16 & $\mathrm{~F}$ & 110 & 6 & 10 & 7 & 9 & 1 \\
\hline 10 & 17 & $\mathrm{~F}$ & 98.5 & 9 & 8 & 9 & 8 & 2 \\
\hline 11 & 13 & $M$ & 118.5 & 6.5 & 6.5 & 5 & 8 & 2 \\
\hline 12 & 17 & $M$ & 103 & 6 & 11 & 2 & 15 & 2 \\
\hline 13 & 14 & $\mathrm{~F}$ & 112.5 & 7 & 7 & 1.5 & 12.5 & 1 \\
\hline 14 & 12 & $M$ & 103.5 & 8 & 4 & 2.5 & 9.5 & 2 \\
\hline 15 & 14 & $\mathrm{~F}$ & 119 & 8 & 6 & 3 & 11 & 2 \\
\hline 16 & 15 & $M$ & 104.5 & 10 & 5 & 3 & 12 & 1 \\
\hline
\end{tabular}

CSL indicates Chinese Sign Language; F, female; HA, hearing aid; M, male; 1, congenital cause of deafness; 2, deafness caused by exposure to ototoxic medication.

metrics) in adolescents were mainly consistent with the changes reported in adults. ${ }^{11}$ Considering the developing brain is both more vulnerable to deprivation and more plastic than the adult brain, we further hypothesized that any brain morphologic alterations should be larger in prelingually deaf adolescents when compared with adults.

To test the hypothesis and to obtain a better understanding of the mechanisms of prelingual deafness, we therefore quantified in greater detail WM developmental abnormalities in adolescents with prelingual deafness by using DTI and tract-based spatial statistics. Then, we correlated the DTI metrics with measures of functional use, namely the duration of experience in sign language and hearing aid use, to investigate the effects of functional use on brain integrity. DTI was used because it is one of the most popular MR imaging techniques in brain research for its high sensitivity in the detection of microstructural alterations. ${ }^{12-14}$ When analyzed with TBSS, DTI studies have the advantages of higher spatial registration and smoothing, thus enabling more accurate results.

\section{MATERIALS AND METHODS \\ Participants}

A total of 16 prelingually deaf adolescents (age at imaging [mean $\pm \mathrm{SD}$ ], $14.56 \pm 2.10$ years, age range: $10-18$ years; 8 boys and 8 girls) and 16 control adolescents matched for age, sex, and right- or left-handedness (age at imaging [mean \pm SD], $14.75 \pm$ 2.38 years; age range: $10-18$ years; 8 boys and 8 girls) were included in the study. All patients became deaf mainly because of drug toxicity or by heredity before age 1 year. The deaf participants experienced sensorineural hearing loss, with the better ear having a mean pure tone average air conduction threshold of $>90$ $\mathrm{dB}$ of hearing loss and no single frequency $>45 \mathrm{~dB}$ hearing loss at $500 \mathrm{~Hz}$ to $4000 \mathrm{~Hz}$. All of the deaf adolescents were trained in Chinese Sign Language for at least 4 years (mean, $7.2 \pm 2.97$ years; range, 4-13 years). The control participants were monolingual Chinese speakers who were healthy with no history of hearing disorder. All of the participants had normal MR imaging findings of the brain and no history of neurologic or psychiatric illness such as meningitis, cytomegalovirus, and traumatic injury. After
Table 2: Demographic data of control children

\begin{tabular}{llc}
\hline Control No & Sex & Age (y) \\
\hline 1 & M & 13 \\
2 & M & 16 \\
3 & F & 16 \\
4 & M & 16 \\
5 & M & 16 \\
6 & M & 16 \\
7 & F & 10 \\
8 & F & 11 \\
9 & F & 18 \\
10 & F & 13 \\
11 & M & 11 \\
12 & $F$ & 16 \\
13 & M & 17 \\
14 & F & 16 \\
15 & F & 16 \\
16 & M & 15 \\
\hline
\end{tabular}

the institutional review board approved our study, written informed consent was obtained from each participant according to the Declaration of Helsinki. The detailed patient data are shown in Table 1, along with the data of the control group in Table 2.

\section{MR Imaging Acquisition}

Diffusion tensor imaging was acquired on a 3T Signa HDxt MR imaging scanner (GE Healthcare, Milwaukee, Wisconsin) by use of an 8-channel phased-array head coil. The protocol was as follows: spin-echo, echo-planar imaging with a spatial resolution of $1 \times 1 \times 2 \mathrm{~mm}, 2$-mm section thickness, no intersection gap, TR of $17,000 \mathrm{~ms}$, TE of $96 \mathrm{~ms}$, field of view of $24 \times 24 \mathrm{~cm}$, and imaging matrix of $256 \times 256$. Motion-probing gradients were applied along 15 noncollinear directions with a b factor of $1000 \mathrm{~s} / \mathrm{mm}^{2}$ after an acquisition without diffusion weighting $\left(b=0 \mathrm{~s} / \mathrm{mm}^{2}\right)$. T1-weighted images were acquired by use of a spoiled gradientecho sequence with the following parameters: flip angle, $13^{\circ}$; TR, $9 \mathrm{~ms}$; TE, $3.5 \mathrm{~ms}$; inversion time, $450 \mathrm{~ms}$; section thickness, $1 \mathrm{~mm}$; acquisition matrix, $256 \times 256$; and field of view, $24 \mathrm{~cm} \times 24 \mathrm{~cm}$. We obtained 196 contiguous sagittal sections with a voxel size of $1 \times 1 \times 1 \mathrm{~mm}^{3}$. 


\section{DTI Data Processing and TBSS}

After the image acquisition, we used the Oxford Centre for Functional MRI of the Brain Diffusion Toolbox (FDT 2.0) within FSL v4.1 (http://www.fmrib.ox.ac. $\mathrm{uk} / \mathrm{fsl}$ ) to transform the diffusion images to NIfTI format on a workstation for further processing. For each participant, 15 DTI volumes with a b-value of 1000 $\mathrm{s} / \mathrm{mm}^{2}$ were first affine-registered to the b0 volume for correction of eddy current distortion and simple head motion. The next step was to remove nonbrain tissue pixels from the native b0 image by using the Brain Extraction Tool ${ }^{15}$ of FSL; a fractional intensity threshold of 0.3 was selected, resulting in a nonbrain tissue-extracted $4 \mathrm{D}$ image and a binary brain mask for each particpant. We then used the eddy-corrected 4D data and corresponding brain mask to fit the diffusion tensor model at each voxel by using the FMRIB Diffusion Toolbox. ${ }^{15}$ This step generates maps of fractional anisotropy, mean diffusivity, and 3 eigenvalues $\left(\lambda_{1}, \lambda_{2}, \lambda_{3}\right) . \lambda_{1}$ is also called axial diffusivity. We then calculated the map of the radial diffusivity (perpendicular eigenvalue, $\lambda_{23}$ ) by averaging the maps of $\lambda_{2}$ and $\lambda_{3}$.

The standard TBSS procedure was then applied to the data. The FA images from each participant were co-registered to the predefined FMRIB58_FA standard-space image provided by FSL by use of nonlinear registration, and affinealigned into Montreal Neurological Institute 152 standard space. The resulting standard-space FA images of each participant were averaged to create a mean FA image, which was then fed into an FA skeletonization program to create a skeleton image of WM tracts; a threshold of 0.2 was selected to define the border of WM and gray matter. Then, each participant's local maximal FA intensity along the perpendicular direction of the WM tract was projected to the mean FA skeleton to carry out the voxelwise statistics across each participant. The same projection method was applied to the MD, axial diffusivity $\left(\lambda_{1}\right)$, and radial diffusivity images.

We carried out voxelwise cross-participant statistics on the skeleton image by using FSL's Randomize tool, which uses a permutation-based statistical inference that does not rely on a Gaussian distribution. ${ }^{16}$ Random Monte Carlo-simulated samples of 10,000 permutations were used as null distribution. Group comparisons between deaf adolescents and healthy control adolescents were then performed. Clusters with sizes of $>20$ voxels and $P$ value of $<.05$ (corrected for multiple comparisons) were identified as significant, according to the threshold-free cluster enhancement method, ${ }^{17}$ to avoid definition of an initial cluster-
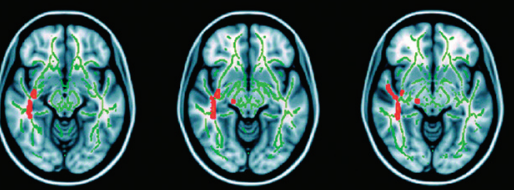

$Z=-8$

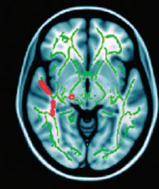

$z=-6$

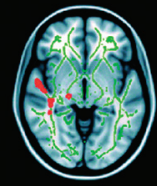

$z=-4$
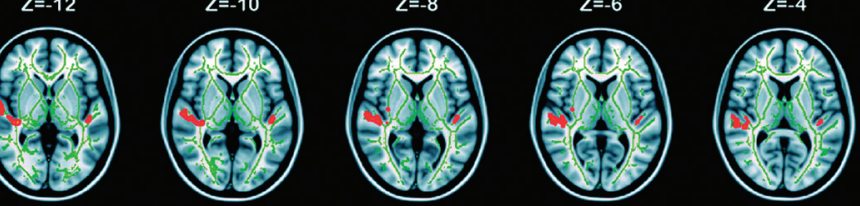

$\mathrm{Z}=0$

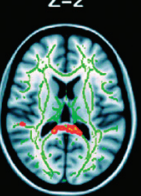

$\mathrm{Z}=4$

$\mathrm{Z}=6$

$\mathrm{Z}=8$
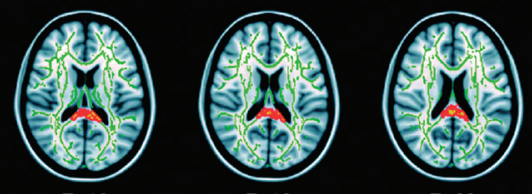

$\mathrm{Z}=14$
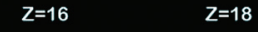

$\mathrm{Z}=20$
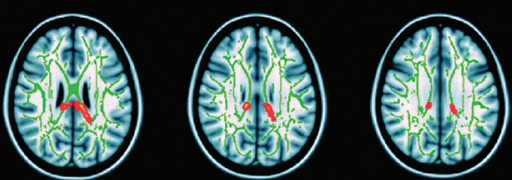

$Z=30$

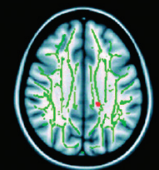

$\mathrm{Z}=32$

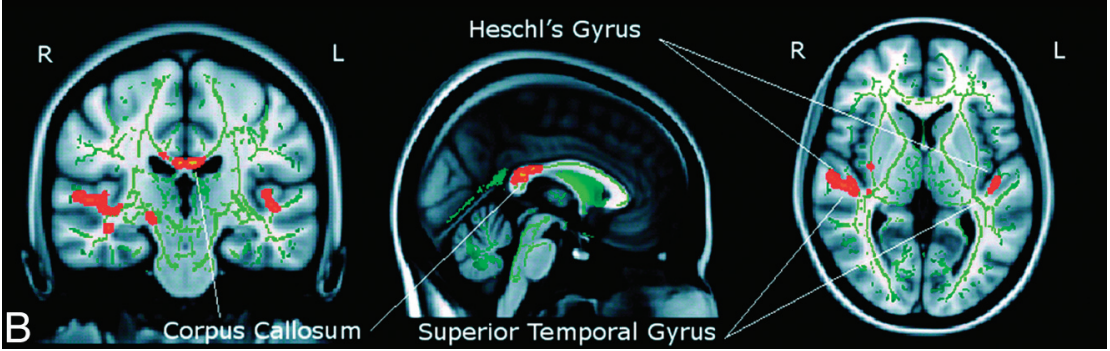

FIG 1. $A$, The identified FA skeleton clusters at $P<.05$ (corrected for multiple comparisons) were filled (by use of tbss_fill script implemented in FSL) to make the presentation easy to MNI T1 1-mm template and the FA skeleton (green). Red-yellow voxels represent regions in which FA was significantly decreased in prelingually deaf children relative to healthy control children. Axial sections from $Z=-14$ to 32 in the MNI coordinate are shown. $B, 3 D$ view of the identified FA skeleton clusters ( $X=90 ; Y=103$; and $Z=76$ ).

forming threshold or carrying out a large amount of data smoothing. To find out whether the duration of auditory experience was related to brain anisotropy, we calculated the Pearson correlation to relate FA, MD, and RD values of HG and STG with biographic data of sign language and hearing aid use, using SPSS v16.0 (SPSS, Chicago, Illinois). A $P$ value of $<.05$ was regarded as significant in the correlation analysis.

\section{RESULTS}

Compared with healthy control adolescents, prelingually deaf adolescents showed significant FA reductions of multiple WM skeleton clusters (Fig 1A). These regions were bilateral WM in the superior temporal gyrus, Heschl gyrus, and planum polare. In addition, decreased FA was also seen in the splenium of the corpus callosum and right thalamus (Fig $1 B$ ). The coordinates of the local maxima and cluster size are listed in Table 3. We did not find any significant FA increases compared with the healthy control participants. 
Table 3: Skeleton clusters showing significantly decreased FA at $P<.05$

\begin{tabular}{|c|c|c|c|c|c|c|}
\hline \multirow[b]{2}{*}{ Cluster Index } & \multirow[b]{2}{*}{ Voxels } & \multirow[b]{2}{*}{$P$ Value } & \multicolumn{3}{|c|}{ MNI Coordinates (mm) } & \multirow[b]{2}{*}{ Anatomic Region } \\
\hline & & & $\mathbf{X}$ & $\mathbf{Y}$ & Z & \\
\hline 1 & 619 & .027 & -5 & -27 & 23 & Corpus callosum \\
\hline 2 & 617 & .028 & 52 & -7 & -6 & R STG, R HG, R PP \\
\hline 3 & 73 & .030 & -47 & -23 & -1 & L STG, L HG, L PP \\
\hline 4 & 36 & .030 & 36 & -14 & -13 & R external capsule \\
\hline 5 & 24 & .030 & 20 & -20 & -6 & $\mathrm{R}$ thalamus \\
\hline
\end{tabular}

L indicates left; MNI, Montreal Neurological Institute; PP, planum polare; R, right.

Table 4: Skeleton clusters showing significantly increased RD at $P<.05$

\begin{tabular}{lcccccc} 
& & \multicolumn{3}{c}{ MNI Coordinates $(\mathbf{m m})$} & $\mathbf{Z}$ & Anatomic Region \\
\cline { 3 - 6 } Cluster Index & Voxels & $\boldsymbol{P}$ Value & $\mathbf{X}$ & $\mathbf{Y}$ & $\mathbf{Z}$ & R STG, R HG, R PP \\
\hline 1 & 2627 & 0.041 & 53 & 95 & 95 & Corpus callosum \\
2 & 2213 & 0.041 & 134 & -103 & 76 & L STG, L HG, L PP \\
\hline
\end{tabular}

$\mathrm{R}$ indicates right; L, left; PP, planum polare.

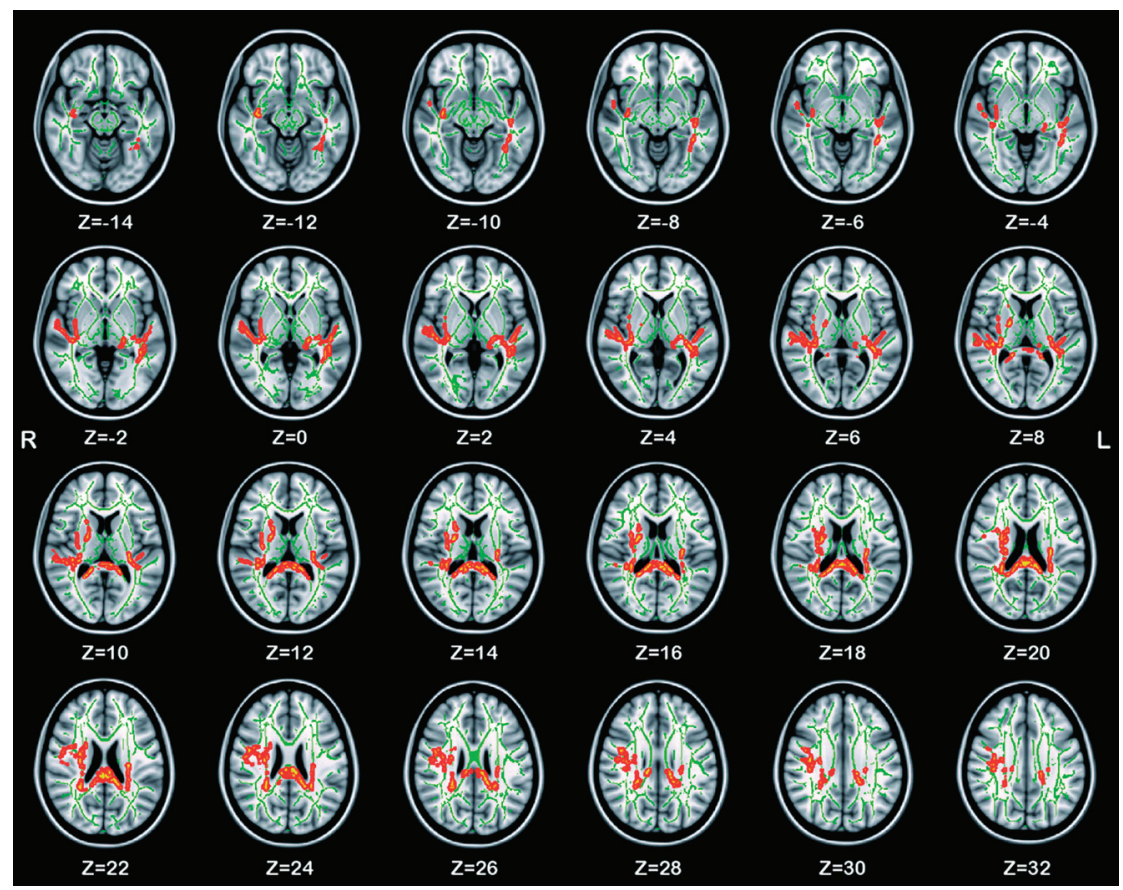

FIG 2. White matter structures showing significantly increased RD in prelingually deaf children (corrected for multiple comparisons; $P<.05$ ), filled by use of the tbss_fill script of FSL to aid visualization. The background image is the standard MNI T1 1-mm template and the FA skeleton (green). Red-yellow voxels represent regions in which RD was increased significantly in prelingually deaf children relative to healthy control children. Axial sections from Z $=-14$ to 32 in the MNI coordinate are shown.

Several RD clusters were significantly increased in prelingually deaf adolescents, as shown in Table 4 and Fig. 2. An RD increase in the bilateral centrum semiovale was also detected, particularly in the right hemisphere. No significant RD decrease was detected.

We found significant FA- and RD-altered areas mainly in the auditory cortex and corpus callosum. The primary auditory cortex is located in the posterior half of the STG. It also dives into HG, which is the first region of the auditory pathway where we expected the most serious anisotropy changes to occur. All of our deaf participants had been using sign language and hearing aids for several years, but the duration of the hearing experience (ie, time of functional use) was rather variable among them. The mean RD value of the right STG in deaf adolescents correlated significantly with the duration of sign language use $(r=-0.518, P=.040$, Table 5, Fig 3), but no significant correlations were found between biographic data (duration of sign language and hearing aid use) and FA of the left HG, right HG, left STG, and right STG (Table 5).

\section{DISCUSSION}

Using DTI, we found multiple structural abnormalities in different brain areas including the bilateral STG, HG, planum polare, corpus callosum, and right thalamus. No correlation was found between DTI metrics and the experience of sign language except in only $1 \mathrm{WM}$ structure, which was a negative correlation. This finding shows that WM anisotropy changes are largely independent of functional use. These findings are partially in agreement with those of previous studies with adults, ${ }^{10,11,18}$ indicating that prelingual deafness in adolescents is mainly consistent with that in adults but shows certain differences.

Compared with other imaging techniques, DTI is more sensitive because it can detect WM microstructural changes well before a macrostructural loss of WM becomes measurable by other procedures, such as voxel-based morphometry. ${ }^{19}$ Although the precise underlying mechanism for the changes of anisotropy is still unclear, decreased FA or increased RD may be interpreted as loss or insufficient development of myelination, loss of axons, gliosis, or tissue edema. Taking into account that our prelingually deaf adolescents did not show any pathologic changes as a result of brain injury, we can ascertain that the anisotropy changes may best be attributed to axonal loss and/or lack of myelination at an early age in life. The specific findings and their relevance are now discussed.

AJNR Am J Neuroradiol 34:1264-70 Jun 2013 www.ajnr.org 
Table 5: Correlation between biographic data and DTI metrics

\begin{tabular}{lclcc}
\hline & Metrics & Area & $\boldsymbol{t}$ & $\boldsymbol{P}$ value \\
\hline Sign language & FA & L HG & 0.61 & .55 \\
& & R HG & 1.17 & .26 \\
& L STG & 1.25 & .23 \\
& R STG & 1.72 & .11 \\
& RD & L HG & -1.11 & .29 \\
& R HG & -1.03 & .32 \\
& L STG & 0.04 & .97 \\
Hearing aids & R STG & -2.26 & $.04^{*}$ \\
& & L HG & 1.25 & .23 \\
& & R HG & 0.03 & .98 \\
& & L STG & 0.83 & .42 \\
& & R STG & 1.44 & .17 \\
& RD & L HG & -0.03 & .98 \\
& & R HG & 0.97 & .35 \\
& L STG & 0.81 & .43 \\
& & R STG & -0.68 & .51 \\
& & &
\end{tabular}

L indicates left; $R$, right.

* indicates statistically significant.

\section{FA Decrease in STG and HG}

Our main finding was decreased fractional anisotropy in the WM regions located in or near the auditory cortex, specifically bilateral changes in the STG and HG, which are consistent with previous studies. ${ }^{10,11}$ The primary auditory cortex is believed to be involved in most of the high-level processing that takes place in sound processing and is essential for recognizing when sound starts, stops, or changes pitch. ${ }^{20,21}$ The FA loss is more significant in the right STG and HG than in the left hemisphere, and the significant decreased FA areas in or near the right STG and HG are much greater than on the left side. ${ }^{4}$ FA is a DTI image-derived parameter that characterizes the intrinsic feature of tissue microstructure and microdynamics. It depends on the relative strength of axial diffusivity and RD. A decrease of axial diffusivity may be

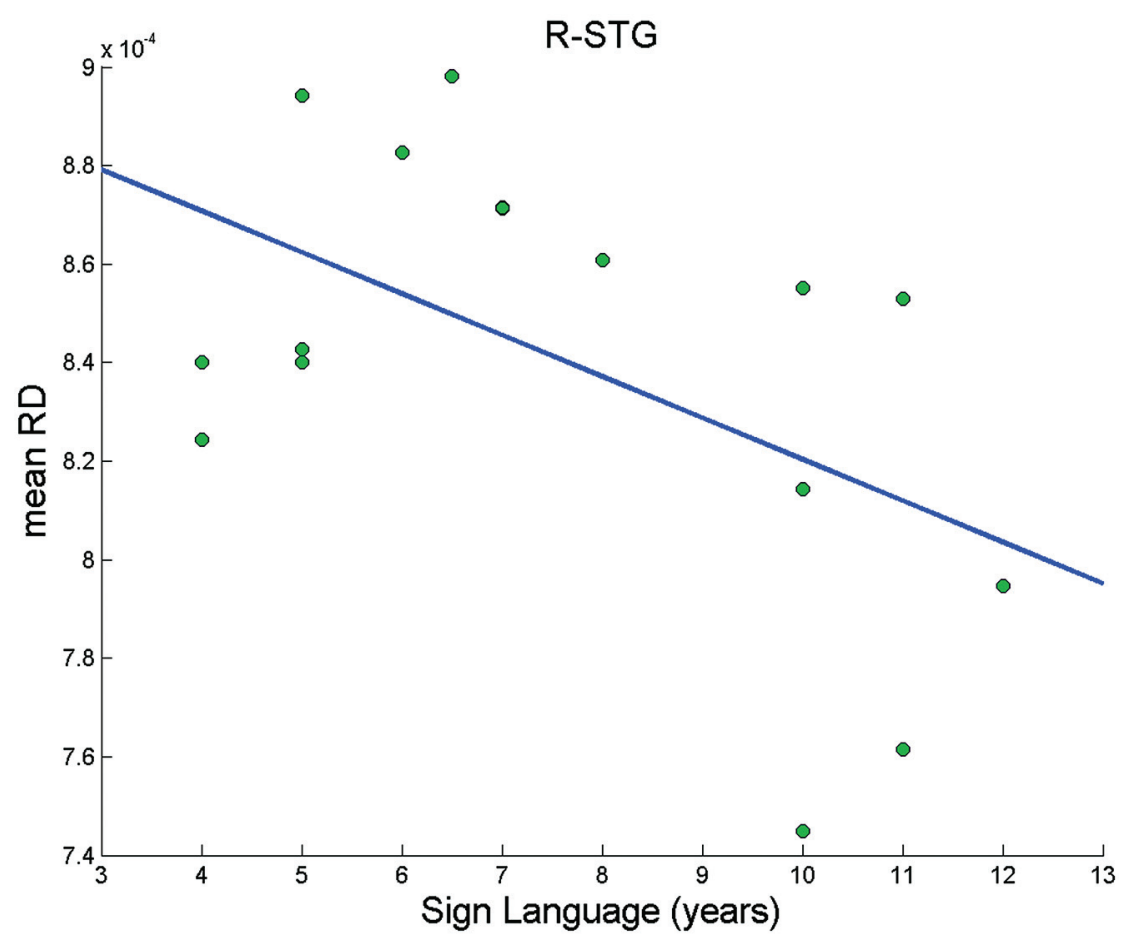

FIG 3. Significant correlation between duration of sign language use and RD of the right STG, $r=-0.518 ; P=.040$. indicative of axonal damage in animal models, whereas an RD increase is related to demyelination. Both changes will result in FA decrease. $^{22}$ The findings here may be related to the widely validated conclusion that the right auditory cortex is more sensitive to tonality, ${ }^{5,23}$ which is consistent with the study by Kim et al. ${ }^{11}$

\section{FA Decrease in Planum Polare and Thalamus}

A significant FA decrease was found in the planum polare, which is part of the secondary auditory cortex. Magnetoencephalography ${ }^{24}$ and functional MR imaging studies ${ }^{25}$ revealed that this area is possibly involved in early syntactic parsing processes, especially in grammatical processes. In addition, a positron-emission tomography study ${ }^{26}$ has demonstrated that repetition and harmonization can activate the planum polare of amateur musicians, and this area is highly active when a professional pianist performs a J.S. Bach concerto blindfolded, ${ }^{27}$ indicating that this area supports higher musical representations. ${ }^{25} \mathrm{~A}$ recent functional MR imaging study ${ }^{28}$ indicates that the planum polare of a neonate, whose brain was not yet functionally specialized, was activated by musical stimuli. For adults, this area becomes increasingly involved with increasing melodic complexity. ${ }^{29}$ Thus, the decreased FA may indicate that the syntactic parsing, pitch, and melody processing in prelingually deaf adolescents are left underdeveloped because of the early lack of experience and exposure to spoken language and music. Nearly all incoming information to the cortex is routed through deep gray matter structures in the thalamus. ${ }^{30}$ Specifically, the medial geniculate nucleus of the thalamus is an obligatory relay for all ascending auditory information destined to reach the primary auditory cortex. MR imaging studies $^{31-33}$ have shown that the thalamus, especially the right thalamus, plays an important role in auditory signal processing. ${ }^{34}$ The FA decrease in the right thalamus indicates that it is vital for auditory processing and is hampered in development because of a lack of auditory input in early age.

\section{FA Decrease in the Corpus Callosum}

The corpus callosum is the largest commissural WM pathway connecting both hemispheres, allowing for communication and coordination to integrate motor, sensory, and cognitive activities on both sides of the brain. Axons in the most caudal region of the corpus callosum-the splenium - connect the occipital, temporal, and parietal regions. ${ }^{35}$ The FA decreases we found in the posterior part of the corpus callosum are nearly the same as the parcellated areas corresponding to the temporal lobe projections in the study by Huang et al. ${ }^{36}$ However, our finding conflicts with the results of the study by Kim et $\mathrm{al},{ }^{11}$ who observed increased (rather than decreased) FA in the corpus callosum. This discrepancy may be attributed to the younger age of our participants, who may have not had enough visual exposure. The significant $(P<.05)$ FA clus- 
ter decrease was greater in the corpus callosum of the participants from our study vs the corpus callosum in the participants from the study by Li et al. ${ }^{10}$

\section{RD Increase in the Brain}

To further investigate the structural differences between healthy control participants and prelingually deaf patients, we also examined other diffusion parameters such as axial diffusivity, $\mathrm{MD}$, and $\mathrm{RD}$. We found a significant difference in RD between groups, and this change showed a large degree of overlap of the FA clusters. The difference of axial diffusivity and MD between groups, however, fell below statistical significance. The RD increased, but the FA decreased in multiple areas, suggesting increased water mobility perpendicular to the axons. It is interesting to note that we detected a significant negative relationship $(r=-0.518 ; P=$ .040) between the duration of sign language and the RD of the right STG (Fig 3). This finding could indicate that sign language may have a positive effect on myelination of the right STG.

\section{Study Limitations}

Despite these interesting findings, our study had some limitations. The number of participants was relatively small for correlations of WM integrity and DTI metrics. Also, we did not consider the potential effects of some clinical information such as education and intelligence levels, both of which may have an impact on brain anisotropy. In addition, DTI has 2 major limitations: the inability to resolve multiple fiber orientations inside of the same voxel, and the lack of specificity of DTI indices (ie, the paradox of white matter integrity). ${ }^{37}$ Thus, additional studies are needed to substantiate our conclusions.

\section{CONCLUSIONS}

Compared with healthy control adolescents, prelingually deaf adolescents showed decreased FA and increased RD in multiple areas including the STG, HG, planum polare, and corpus callosum. The results support our hypothesis that changes were mainly located in the auditory-associated brain areas and that the affected areas in prelingually deaf adolescents were larger than those previously reported in adults by other laboratories. ${ }^{10}$ Changes in anisotropy might be attributed to axonal loss or shrinkage and/or lack of myelination, which has a major effect on the life of the growing adolescent. The FA and RD changes that we have observed are broadly consistent with previous studies of deaf adults. ${ }^{10,11}$ However, we found that only 1 anisotropy change in the auditory cortex - the RD increase in the right STG-correlated with duration of sign language use and none correlated with hearing aid use. From this finding, we conclude that WM anisotropy alterations in prelingually deaf adolescents are largely independent of functional use.

\section{ACKNOWLEDGMENTS}

We thank all of the adolescents and their families for their participation in the study. Special acknowledgments go to Wen Song and Xihong Liang for their help in recruiting participants of the study and looking after them.

\section{REFERENCES}

1. Kral A, O’Donoghue GM. Profound deafness in childhood. $N$ Engl J Med 2010;363:1438-50

2. Gilley PM, Sharma A, Mitchell TV, et al. The influence of a sensitive period for auditory-visual integration in children with cochlear implants. Restor Neurol Neurosci 2010;28:207-18

3. Sadato N, Okada T, Honda M, et al. Cross-modal integration and plastic changes revealed by lip movement, random-dot motion and sign languages in the hearing and deaf. Cereb Cortex 2005; 15 : 1113-22

4. Meyer M, Toepel U, Keller J, et al. Neuroplasticity of sign language: implications from structural and functional brain imaging. Restor Neurol Neurosci 2007;25:335-51

5. Smith KM, Mecoli MD, Altaye M, et al. Morphometric differences in the Heschl's gyrus of hearing impaired and normal hearing infants. Cereb Cortex 2011;21:991-98

6. Emmorey K, Allen JS, Bruss J, et al. A morphometric analysis of auditory brain regions in congenitally deaf adults. Proc Natl Acad Sci U S A 2003; 100:10049-54

7. Shibata DK. Differences in brain structure in deaf persons on MR imaging studied with voxel-based morphometry. AJNR Am J Neuroradiol 2007;28:243-49

8. Li J, Li W, Xian J, et al. Cortical thickness analysis and optimized voxel-based morphometry in children and adolescents with prelingually profound sensorineural hearing loss. Brain Res 2012;1430: $35-42$

9. Sharma A, Dorman MF, Spahr AJ. A sensitive period for the development of the central auditory system in children with cochlear implants: implications for age of implantation. Ear Hear 2002;23: 532-39

10. Li Y, Ding G, Booth JR, et al. Sensitive period for white-matter connectivity of superior temporal cortex in deaf people. Hum Brain Mapp 2012;33:349-59

11. Kim DJ, Park SY, Kim J, et al. Alterations of white matter diffusion anisotropy in early deafness. Neuroreport 2009;20:1032-36

12. Basser PJ, Pierpaoli C. Microstructural and physiological features of tissues elucidated by quantitative-diffusion-tensor MRI. J Magn Reson B 1996;111:209-19

13. Huppi PS, Dubois J. Diffusion tensor imaging of brain development. Semin Fetal Neonat Med 2006;11:489-97

14. Basser PJ, Mattiello J, LeBihan D. MR diffusion tensor spectroscopy and imaging. Biophys J 1994;66:259-67

15. Smith SM. Fast robust automated brain extraction. Hum Brain Mapp 2002;17:143-55

16. Nichols TE, Holmes AP. Nonparametric permutation tests for functional neuroimaging: a primer with examples. Hum Brain Mapp 2002;15:1-25

17. Smith SM, Nichols TE. Threshold-free cluster enhancement: addressing problems of smoothing, threshold dependence and localisation in cluster inference. Neuroimage 2009;44:83-98

18. Wu CM, Ng SH, Wang JJ, et al. Diffusion tensor imaging of the subcortical auditory tract in subjects with congenital cochlear nerve deficiency. AJNR Am J Neuroradiol 2009;30:1773-77

19. Douaud G, Jbabdi S, Behrens TE, et al. DTI measures in crossingfibre areas: increased diffusion anisotropy reveals early white matter alteration in MCI and mild Alzheimer's disease. Neuroimage 2011;55:880-90

20. Zatorre RJ, Belin P, Penhune VB. Structure and function of auditory cortex: music and speech. Trends Cogn Sci 2002;6:37-46

21. Zatorre RJ, Belin P. Spectral and temporal processing in human auditory cortex. Cereb Cortex 2001;11:946-53

22. Scheel M, Prokscha T, Bayerl M, et al. Myelination deficits in schizophrenia: evidence from diffusion tensor imaging. Brain Struct Funct 2013;218:151-56

23. Zatorre RJ, Evans AC, Meyer E, et al. Lateralization of phonetic and pitch discrimination in speech processing. Science 1992;256:846-49

24. Friederici AD, Wang Y, Herrmann CS, et al. Localization of early 
syntactic processes in frontal and temporal cortical areas: a magnetoencephalographic study. Hum Brain Mapp 2000;11:1-11

25. Meyer M, Friederici AD, von Cramon DY. Neurocognition of auditory sentence comprehension: event related fMRI reveals sensitivity to syntactic violations and task demands. Cognitive Brain Res 2000;9:19-33

26. Brown S, Martinez MJ, Hodges DA, et al. The song system of the human brain. Cognitive Brain Res 2004;20:363-75

27. Parsons LM, Sergent J, Hodges DA, et al. The brain basis of piano performance. Neuropsychologia 2005;43:199-215

28. Perani D, Saccuman MC, Scifo P, et al. Functional specializations for music processing in the human newborn brain. P Natl Acad Sci U S A 2010;107:4758-63

29. Patterson RD, Uppenkamp S, Johnsrude IS, et al. The processing of temporal pitch and melody information in auditory cortex. Neuron 2002;36:767-76

30. Behrens TE, Johansen-Berg $\mathrm{H}$, Woolrich MW, et al. Non-invasive mapping of connections between human thalamus and cortex using diffusion imaging. Nat Neurosci 2003;6:750-57

31. Versino M, Beltrami G, Uggetti C, et al. Auditory saccade impair- ment after central thalamus lesions. J Neurol Neurosurg Psychiatry 2000;68:234-37

32. Schönwiesner M, Krumbholz K, Rübsamen R, et al. Hemispheric asymmetry for auditory processing in the human auditory brain stem, thalamus, and cortex. Cereb Cortex 2007;17:492-99

33. Devlin JT, Sillery EL, Hall DA, et al. Reliable identification of the auditory thalamus using multi-modal structural analyses. Neuroimage 2006;30:1112-20

34. Shergill SS, Brammer MJ, Williams SCR, et al. Mapping auditory hallucinations in schizophrenia using functional magnetic resonance imaging. Arch Gen Psychiatry 2000;57:1033-38

35. Witelson SF. Hand and sex differences in the isthmus and genu of the human corpus callosum. A postmortem morphological study. Brain 1989;112:799-835

36. Huang H, Zhang J, Jiang H, et al. DTI tractography based parcellation of white matter: application to the mid-sagittal morphology of corpus callosum. Neuroimage 2005;26:195-205

37. Dell'Acqua F, Catani M. Structural human brain networks: hot topics in diffusion tractography. Curr Opin Neurol 2012;25: 375-83 